\title{
Klinisches Risikomanagement am Ospidal d'Engiadina Bassa
}

\section{Joachim Koppenberg ${ }^{a}$, Hans-Peter Sinniger ${ }^{b}$, Peter Gausmann}

a Dr. med., Chefarzt Anästhesiologie, Ospidal d'Engiadina Bassa, Scuol

b Lic. iur., Direktor Ospidal d'Engiadina Bassa, Scuol

c Dipl. Kfm., Dipl.-Pflg.-Päd. Peter Gausmann, Gesellschaft für Risiko-Beratung $\mathrm{mbH}$ Detmold, D*

* Interessenverbindung: Prokurist der Gesellschaft für Risiko-Beratung.
Korrespondenz:

Dr. med. Joachim Koppenberg Ospidal d'Engiadina Bassa

CH-7550 Scuol

Tel. 0818611000

Fax 0818649904

joachim.koppenberg@

ospidal-scuol.ch

\section{Einleitung}

Die Qualität der medizinischen Versorgung in den Spitälern wurde in den letzten 10 bis 15 Jahren durch vielfältige externe und insbesondere interne Massnahmen ständig verbessert. Dies ist notwendig in Zeiten der Ökonomisierung, Rationalisierung, der Fallzahlerhöhung und Verweildauerverkürzung. Gegenläufig zu dieser Qualitätsoffensive bewegt sich allerdings die Anzahl vermuteter Behandlungsfehler auf einem Rekordniveau. Dies ist in der Schweiz nicht anders als im europäischen Umland [1]. In unseren Spitälern werden zunehmend aufgeklärte und kritische Patienten behandelt, welche die Ergebnisse der modernen Medizin nicht schicksalhaft hinnehmen, die ihre Erwartungen an Medizin und Pflege artikulieren und die eine vermeintliche Schlechtleistung reklamieren.

Behandlungsfehlervorwürfe, so zeigen die Statistiken, sind häufig ungerechtfertigt - auf seit Jahren gleichbleibendem Niveau gehen etwa ein Drittel der Forderungen in die Regulierung, zwei Drittel werden ohne Haftung zu den Akten gelegt [2]. Gleichwohl ist jeder angezeigte Vorwurf mit zeitaufwendigen Recherchen, Anhörungen und mit Darlegungsaufwand für den Angeschuldigten verbunden. Besonders tragisch ist es für ein Spital, wenn eine letztlich unbegründete Behandlungsfehlervermutung den Medien zugespielt wird. Dann kann in kürzester Zeit die langjährige Arbeit zum Aufbau eines positiven Images einer Abteilung zunichte gemacht werden.

Häufig wird mit dem Begriff klinisches Risikomanagement in erster Linie ein funktionierendes Fehlermeldesystem (CIRS = critical incident reporting) in Verbindung gebracht [3-5]. Ein systematisches Risikomanagement ist jedoch umfassender und betrachtet Strukturen, Prozesse und Ergebnisse medizinisch-pflegerischen Handelns aus dem Blickwinkel früherer Schäden in vergleichbaren Spitälern. Die Gesellschaft für Risiko-Beratung (GRB) aus Detmold/D wertet seit 1994 reale Schadensfälle aus Spitälern in Deutschland, Österreich und der Schweiz aus und leitet hieraus Risiken und geeignete Präventionsmassnahmen ab. Das Wissen aus über
60000 stattgehabten Schadenereignissen wird analysiert und prospektiv zur Verfügung gestellt [6]. Professionelle Risikoberater verfügen somit über einen reichhaltigen Erfahrungsschatz aus Schadenereignissen anderer vergleichbarer Spitäler: Erfahrungen, die sie den Mitarbeitenden der Klinik kommunizieren und auf diese Weise

\section{Résumé}

Pendant une hospitalisation, la sécurité du patient est l'objectif le plus important, outre la réussite du traitement. Malgré la mise en place de plus en plus fréquente de systèmes de gestion de la qualité dans les hôpitaux, le nombre de fautes de traitement présumés ne cesse d'augmenter. En parallèle, le nombre de reproches dûment fondés reste constant.

L'Ospidal d'Engiadina Bassa a, dans le cadre d'un projet pilote, soumis les processus hospitaliers à une analyse externe de gestion du risque. Le programme d'analyse utilisé se base sur une évaluation de plus de 60000 cas de lésions corporelles enregistrés en Suisse, en Autriche et en Allemagne.

Durant plusieurs jours, des experts externes du risque ont analysé la prescription de médicaments, l'organisation des salles d'opération ou la prévention des chutes dans les services de chirurgie, médecine interne, anesthésiologie/médecine du sauvetage et gynécologie/obstétrique ainsi que de paraplégie, qui est un domaine à risque. Ils ont accordé une attention particulière aux soins, à la documentation, à l'information au patient et à organisation.

Le présent article décrit, à l'aide d'exemples, le processus de la gestion théorique du risque ainsi que son application pratique à l'Ospidal d'Engiadina Bassa. 
multiplizieren. Die alltäglichen Betriebsabläufe in Diagnostik, Therapie und Pflege werden mit neutralem Blick auf das Sicherheitsniveau beobachtet und können optimiert werden. Die bisher unauffällige Schadenbilanz am Spital Unterengadin bot keinen konkreten Anlass. Die Motivation der Spitalleitung für dieses Pilotprojekt begründet sich in einem hohen Risikobewusstsein.

\section{Der Risiko-Management-Prozess}

Der Prozess für den Aufbau und die Etablierung eines Risikomanagements (RM) setzt sich in einem Regelkreis aus 4 Schritten zusammen (Abb. 1). Einer Phase der Risikoidentifizierung folgt die Risikobewertung. Hieran schliesst sich die Phase der Risikobewältigung mit der Durchführung geeigneter Massnahmen zur Optimierung des Sicherheitsniveaus an. Sind nach Durchführung dieser Schritte Risiken bekannt und reorganisiert, folgt die Phase der längerfristigen Risikoüberwachung durch Festlegung geeigneter Prüfparameter zur Kontrolle und Steuerung (Stichwort: CIRS). Im Wesen entspricht dieser RM-Prozess dem aus der Qualitätslehre bekannten PDCA-Zyklus.

In der Phase der Risiko-Identifizierung werden die für den medizinischen Schwerpunkt der jeweiligen Klinik bzw. einzelner Fachabteilungen wesentlichen, realen und bedrohlichen Risiken definiert. In der Anästhesie sind dies beispielsweise Risiken der unerkannten Fehlintubation,

\section{Abbildung 1}

Der Risiko-Management-Prozess.

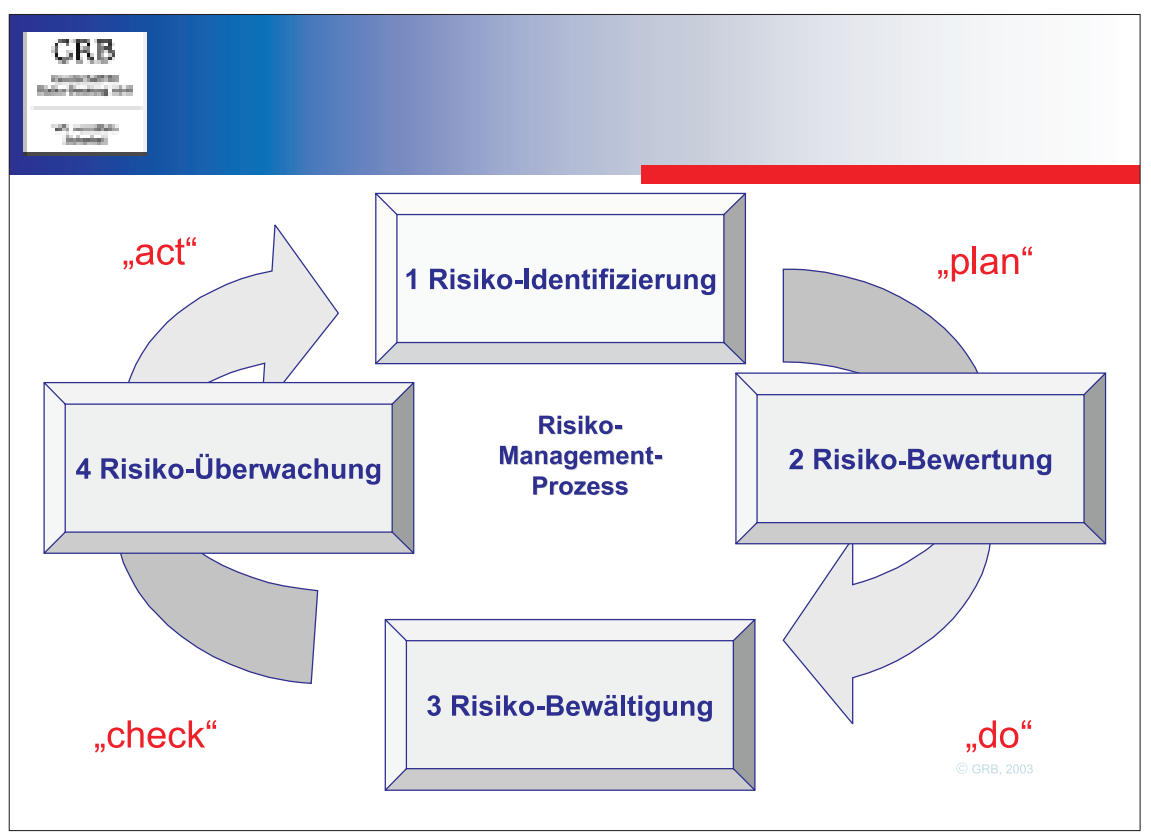

in der Orthopädie das Risiko der Seitenverwechslung oder in der Onkologie die Fehlapplikationen von Chemotherapeutika - um nur einige spezifische, aus Schadenerfahrung ableitbare Risiken zu benennen. Das Bedrohungspotential misst sich an der durch die Risiken bestehenden Gefährdung des Versorgungsauftrags. Eine Risikoidentifizierung setzt Aufmerksamkeit, Bewusstsein und Sensibilisierung aller beteiligten Akteure in einem therapeutischen Team voraus und ist damit nicht nur Aufgabe der Ärzte, sondern ebenso der Pflege- und Assistenzberufe. Den Mitarbeitenden sind die Gefährdungspotentiale in ihrem unmittelbaren Arbeitsbereich in der Regel durch Ausbildung, Weiterbildung und nicht zuletzt durch ihre Berufserfahrung bekannt. Es tritt allerdings im Umgang mit Gefahren ein gewisser Gewöhnungsprozess ein, der Risiken, insbesondere wenn sie sich in der Vergangenheit nicht als Schaden verwirklicht haben, beherrschbar erscheinen lässt. Daher ist es wichtig, die Phase der Risikoidentifizierung für eine vorbehaltlose und selbstkritische Reflexion zu nutzen. Diese darf nicht durch hierarchische Strukturen oder andere Störfaktoren beeinflusst werden.

Die Phase der Risiko-Bewertung dient der Quantifizierung einer möglichen Auswirkung identifizierter Risiken. Das Ausmass eines Risikos wird durch die Bewertung der Wahrscheinlichkeit des Auftretens und der Höhe des damit verbundenen potentiellen Schadens eingeschätzt und z.B. in einem einfachen zweidimensionalen Risiko-Portfolio-Modell dargestellt (Abb. 2). Das Spannungsfeld zwischen Schadenpotential und Eintrittswahrscheinlichkeit markiert den Handlungsbedarf für das therapeutische Team. Die moderierte Bewertung identifizierter Risiken setzt einen fruchtbaren Interaktionsprozess in Gang, an dessen Ende häufig bereits praktikable Lösungsvorschläge erarbeitet werden können.

In der Phase 3 des RM-Prozesses bestehen die Aufgaben der Risiko-Bewältigung darin, aus den zuvor erfassten und bewerteten Risiken Konsequenzen zu ziehen.

Als Bewältigungsstrategien kommen vielfältige und in Zusammenhang mit der Risikoidentifizierung und -bewertung definierte Massnahmen zum Tragen (siehe folgende Beispiele 1-4).

Die Phase 4 der Risiko-Überwachung schliesst den RM-Prozess und stabilisiert ihn für die $\mathrm{Zu}$ kunft. Das Spital installiert und etabliert in dieser Phase ein System zur Erfassung und Analyse von Zwischenfällen und Beinaheschäden im Sinne eines Critical Incident Reporting System (CIRS). 
Abbildung 2

Risiko-Portfolio.

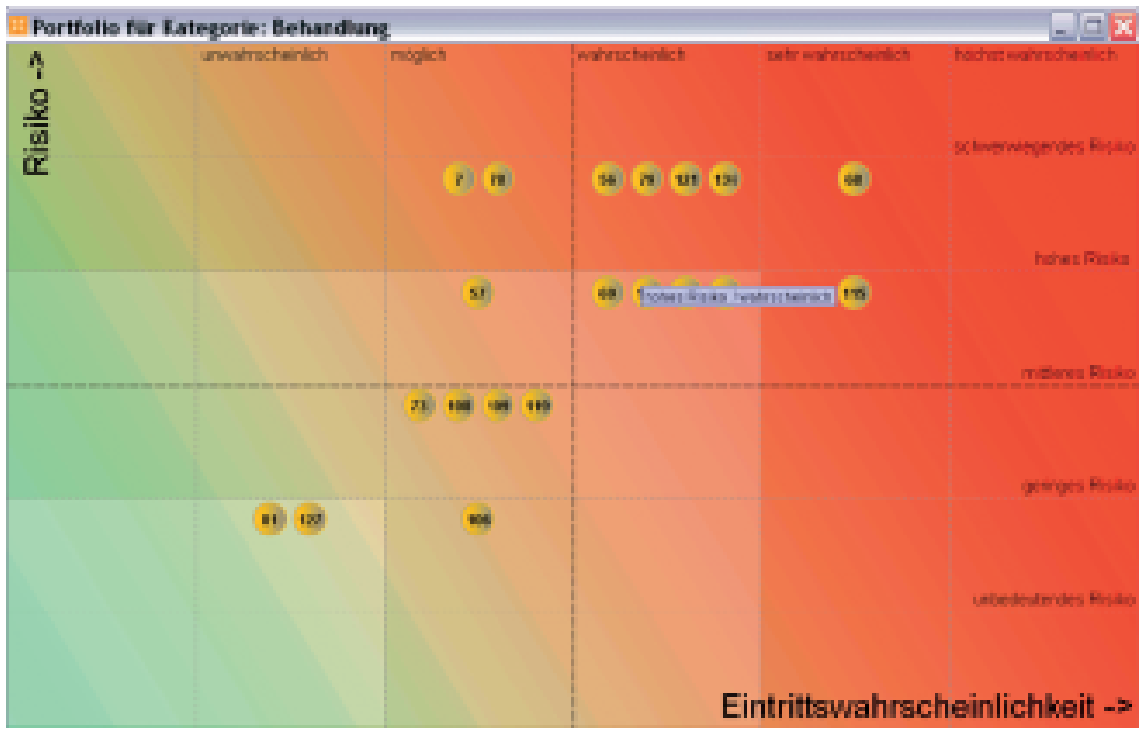

** Diese Tabelle ist in der gedruckten Fassung nicht wiedergegeben, sie findet sich aber im Anhang der Onlineversion des Artikels, die im Internet unter folgender Adresse aufgerufen werden kann: www.saez.ch $\rightarrow$ Archiv $\rightarrow 2006 \rightarrow$ Nr. 34

\section{Risiko-Management-Projekt am OEB}

Das Ospidal d'Engiadina Bassa geht mit dem Aufbau eines umfassenden klinischen RM-Systems neue Wege. Im Rahmen eines Pilotprojektes im Juni 2005 wurden die Fachabteilungen des Spitals auditiert, indem die Mitarbeitenden eine durch externe Risikoberater der Firma GRB moderierte Risikoselbstbewertung vornahmen. Vier zentrale Themen - die Dokumentation, die Patientenaufklärung, die Prozessorganisation und die Behandlungsverfahren - stehen im Mittelpunkt der Risikoberatung.

In den Fachabteilungen Chirurgie, Innere Medizin, Anästhesiologie/Rettungsmedizin und Gynäkologie/Geburtshilfe wurde an drei aufeinanderfolgenden Tagen durch teilnehmende Beobachtung der Arbeitsabläufe und durch Interviews der Mitarbeitenden ein Präventions- und Risiko-Profil (PRP) erarbeitet. Die Mitarbeitenden selbst identifizierten und klassifizierten Schwachstellen im Spannungsfeld zwischen Risikorelevanz und Handlungsbedarf. Insbesondere der Dialog über die Risikosituationen, in denen das therapeutische Team im Rahmen der Ergebnispräsentation eintrat, schärft das Risikobewusstsein und leitet notwendige Reorganisationsmassnahmen ein. Das Team selbst verpflichtet sich zum Handeln und legt einen Massnahmenplan fest, der nach Ablauf eines definierten Zeitintervalls evaluiert wird.

\section{Projektablauf}

Eine wichtige Voraussetzung für ein solches Projekt ist die Unterstützung durch Spitalleitung und Kaderangehörige. Als Gründe zur Durchfüh- rung einer externen Analyse sowie zur Einführung eines Risikomanagements seien genannt: 1) Erhöhung der individuellen Patientensicherheit, 2) Erhöhung der Behandlungssicherheit und somit ein grösstmöglicher Schutz der einzelnen Mitarbeitenden vor ungerechtfertigten Anschuldigungen und Forderungen, 3) Prävention möglicher Haftpflichtschadensfälle mit finanziellen Auswirkungen für das Spital, 4) Verhinderung einer negativen Presseberichterstattung über mögliche Zwischenfälle im Spital. Der letzte Punkt spielt für ein kleines Regionalspital wie das Ospidal d'Engiadina Bassa mit geringeren Patientenzahlen und zunehmender Diskussion um Mindestmengen sicherlich eine grössere Rolle als für ein Zentrumsspital.

Es ist aus verständlichen Gründen nicht jedem Spitalmitarbeiter auf Anhieb sympathisch, dass ein externes Analystenteam die eigenen Arbeiten auf Sicherheitslücken hin überprüfen soll. Daher müssen die Ziele und die Sinnhaftigkeit der Analyse bereits vor Projektbeginn aktiv den Mitarbeitenden vermittelt werden, um ungerechtfertigte Widerstände oder gar Ängste zu verhindern. Es muss klar kommuniziert werden, dass es sich um eine Systemanalyse handelt und keine persönlichen Fehler gesucht oder gar geahndet werden sollen. Zudem sollte klar kommuniziert werden, dass bei den Gesprächen mit den Analysten keine Probleme ausgeklammert oder beschönigt werden.

Stellt man konsequent die Erhöhung der Patientensicherheit in den Vordergrund, so kann v.a. beim Pflegepersonal und den Assistenzärzten eine hohe Motivation zur Mitarbeit verzeichnet werden. Die anfänglich noch vereinzelt wahrnehmbare Skepsis bei einigen Mitarbeitern am OEB («Kontrolle», «Überwachung», u. a.) wich im Rahmen der eigentlichen Begehung schnell einer freundlich positiven Grundstimmung. Die Begehung und Begleitung durch die externen Risikoanalysten wurden als echte Bereicherung in einer freundlichen, fachlich kompetenten Atmosphäre erlebt. Einige Mitarbeitende empfanden das Gespräch über ein evtl. heikles sicherheitsrelevantes Problem mit einem neutralen externen Berater sogar als angenehmer und einfacher als mit einem direkten Vorgesetzen. Besonders positiv wurde der Umstand wahrgenommen, dass bereits bestehende Präventionsmassnahmen erfasst und in die Risikobewertung aufgenommen wurden.

\section{Ergebnisse}

Insgesamt wurden die in Tabelle $1^{\text {** }}$ genannten Bereiche untersucht. Die Analyse fokussierte sich neben dem eigentlichen Behandlungsspektrum 
auf die Struktur der medizinisch-pflegerischen Dokumentation und deren Anwendung, die Aufklärungspraxis sowie die Organisation der Arbeitsabläufe. Besonderer Wert wurde hierbei auf die Abstimmung einzelner Leistungseinheiten und die Schnittstellen gelegt. Neben den klassischen Bereichen wie der Chirurgie, der Inneren Medizin, der Anästhesie und Gynäkolgie/ Geburtshilfe wurden auch gefahrenträchtige Querschnittsbereiche, wie z.B. die Arzneimittelgabe, die OP-Organisation oder die Sturzprophylaxe, betrachtet.

Im folgenden sollen zur Verdeutlichung konkrete Beispiele aus den Bereichen Behandlung, Dokumentation, Patientenaufklärung und Organisation vorgestellt werden.

\section{Behandlung: «Schmerztherapie»}

Im Kapitel «Behandlung» des Bereichs «Schmerztherapie» wurde bei der Analyse ein gut eingeführtes, gut dokumentiertes und konsequent angewandtes Schmerzkonzept vorgefunden (Tabelle $2^{* *}$ ). Hier konnte indirekt der Beweis erbracht werden, dass ein bereits 1998 eingeführtes und seitdem ständig optimiertes Qualitätsmangement entsprechende Präventionsmassnahmen bereits hervorgebracht hat und in diesem Bereich keine konkrete Risikokonstellation und somit auch kein akuter Handlungsbedarf gegeben ist. Dieses Beispiel zeigt, dass im Rahmen der Risikoanalyse neben der Suche nach potentiellen Fehlern auch die bereits bestehenden Präventionsmassnahmen ausreichend berücksichtigt werden. Nicht zu unterschätzen ist in diesem Zusammenhang der Effekt, dass die Mitarbeitenden in der Einsicht bestärkt werden, dass die von ihnen gelebten Standards einem hohen Sicherheitsstandard entsprechen.

\section{Dokumentation: «Geburtsverlauf»}

Die Dokumentation nimmt im Rahmen einer Schadensfallbearbeitung die zentrale Bedeutung ein. Bei der Dokumentation des Geburtsverlaufs konnten zwei Schwachstellen identifiziert werden (Tabelle $3^{\star *}$ ): Einerseits fand keine gemeinsame Dokumentation der Kreisssaaldokumentation von Hebamme und Arzt statt, und zudem wurde bei einem möglichen Hebammenwechsel im Rahmen der Geburtsbegleitung der Personenwechsel nicht verzeichnet. Beide in den Empfehlungen geäusserten Dokumentationsmassnahmen waren ohne grossen Aufwand umsetzbar und erhöhen nun die Behandlungssicherheit der involvierten Mitarbeitenden.

\section{Patientenaufklärung: «Allgemeine Patientenaufklärung»}

Eine selbst noch so sorgfältig durchgeführte $\mathrm{Pa}$ tientenaufklärung über medizinische Eingriffe ist im Schadensfall nicht sehr hilfreich, wenn sie nicht ebenso sorgfältig dokumentiert wurde. Die häufigsten Missstände und somit auch Schadenansprüche werden im Zusammenhang mit mangelnder oder fehlender Dokumentation der Patientenaufklärung gefunden. Die Risikoanalyse am OEB zeigte in diesem Bereich einige Unregelmässigkeiten auf (Tabelle $4^{* *}$ ), die empfohlenen Verbesserungen wurden umgehend eingeleitet. So wurden die aktuellen Papieraufklärungsbögen aktualisiert. Demnächst erfolgt die Umstellung auf internetbasierte Aufklärungsbögen, welche dann auch mehrsprachig in der immer aktuellsten Version abrufbar sind.

\section{Organisation:}

«Patientenaufnahme und -entlassung»

Dass auch bereits gut eingeführte Konzepte immer noch optimierbar sind, zeigt die Analyse des Bereiches «Patientenaufnahme und -entlassung». V.a. bei der Entlassung kann es für Patienten extrem hilfreich sein, Merkblätter mit spezifischen Verhaltensregeln ausgehändigt zu bekommen (z.B. Verhalten nach Gipsanlage). Zudem wird damit das Risiko für Patienten wie auch für das Spital minimiert (z.B. Merkblatt «Gipsanlage»: «Bei Schmerzen im Bereich des Gipses ist unverzüglich ein Arzt oder das Spital aufzusuchen»). Die Merkblätter werden für die häufigsten Entlassdiagnosen entwickelt und in das bestehende Entlassmanagement integriert.

\section{Resonanz und Ausblick}

Das Projekt wurde nach anfänglicher Skepsis von allen Mitarbeitenden sehr begrüsst und intensiv mitgetragen. Neben der vertrauensvollen Atmosphäre trug v.a. die im Mittelpunkt der Analyse stehende Erhöhung der Patientensicherheit stark zum Gelingen des Projekts bei. Neben einer weiteren Sensibilisierung für das Thema Patientenund Behandlungssicherheit wurden die Mitarbeitenden darin bestätigt, dass ihr Spital neben den bereits vorhandenen Sicherheitsstandards weiterführende Massnahmen unterstützt. Nebenbefundlich konnte trotz des heiklen Themas «Risiko im Spital» ein positives Presseecho in den lokalen Medien verzeichnet werden. Mit grossem Interesse und Wohlwollen wurde das Projekt durch den aktuellen Haftpflichtversicherer verfolgt. Von einer bereits geplanten, routinemässi- 
gen Erhöhung der Haftpflichtprämie wurde nach Einsicht in den Abschlussbericht Abstand genommen.

Die Spitalleitung des Ospidal d'Engiadina Bassa zeigt sich mit dem Ergebnis der Analyse äusserst zufrieden und wird die Empfehlungen der Risikoanalysten gemäss der gemeinsam ermittelten Prioritätenliste nach und nach umsetzen. Zur kontinuierlichen Analyse der weiteren Risikoentwicklung am OEB wird zeitnah in den Bereichen ein CIRS-System (riskop ${ }^{\odot}$ ) implementiert.

Konsequenterweise beteiligt sich das Spital am schweizweiten Pilot-Projekt CIRRNET (Critical Incident Reporting and Reacting NETwork) der Stiftung für Patientensicherheit in Zusammenarbeit mit der Schweizerischen Gesellschaft für Anästhesiologie und Reanimation und erwartet aus der überregionalen Vernetzung weitere Impulse für die Erhöhung der Patientensicherheit.

Der vollständige Text inklusive Anhang findet sich in der Internetversion dieser Nummer unter www. saez.ch $\rightarrow$ Archiv $\rightarrow 2006 \rightarrow \mathrm{Nr} .34$.

\section{Literatur}

1 Haller U, Welti S, Haenggi D, Fink D. Von der Schuldfrage zur Fehlerkultur in der Medizin. Schweiz Ärztezeitung. 2005;86:1665-1679.

2 Klocke M. Zahl der Schadensfälle nicht gestiegen. Ku-Sonderheft Risk Management. 2005;8:14-15.

3 Staender S. «Incident Reporting» als Instrument zur Fehleranalyse in der Medizin. ZaeFQ. 2001; 95:479-484.

4 Staender S, Kaufmann M, Scheidegger D. Critical Incident Reporting Systems in Anaesthesia. In: Vicent C, de Mol B (editors). Safety in Medicine. Oxford: Pergamon, Elsevier Science; 2000. p. 65-82

5 Frey B, Kehrer B, et al. Comprehensive critical incident monitoring in a neonatal-pediatric intensive care unit: experience with the system approach. Intensiv Care Med. 2000;26:69-74.

6 Gausmann P. Den Schaden nicht vergüten, sondern verhüten. Ku-Sonderheft Risk-Management 2005;8:16-19. 


\section{Anhang}

Tabelle 1

Inhaltsverzeichnis Risikoanalyse.

\begin{tabular}{|ll}
\hline 1 & Behandlung \\
\hline 1.1 & Schmerztherapie \\
\hline 1.2 & Notfallbehandlung \\
\hline 1.3 & Behandlungsstandards \\
\hline 1.4 & Reanimationsmanagement \\
\hline 1.5 & Standards in der Geburtshilfe \\
\hline 1.6 & Behandlung Rettungsdienst \\
\hline 1.7 .1 & Weitere Behandlungsleistungen \\
\hline 2 & Anästhesie \\
\hline 2.1 & Dokumentation \\
\hline 2.2 & Notaufnahme \\
\hline 2.3 & Verlaufsdokumentation \\
\hline 2.4 & Dokumentation des Geburtsverlaufs \\
\hline 2.5 & Dokumentation im Rettungswesen \\
\hline 3 & Patientenaufklärung \\
\hline 3.1 & Patientenaufklärung in der Ambulanz \\
\hline 3.2 & Allg. Patientenaufklärung \\
\hline 3.3 & Aufklärung im Rettungsdienst \\
\hline
\end{tabular}

\section{Organisation}

4.1 Ambulanzbetrieb

4.2 Arzneimittelgabe

4.3 Patientenaufnahme und -entlassung

$4.4 \quad$ Hygienemanagement

4.5 Archivorganisation

4.6 Intensivstation

4.7 Geburtsorganisation

$4.8 \quad$ Wiederaufbereitung und Sterilisation

4.9 Medizintechnik

4.10 Funktionsbereiche

4.11 Organisation im Rettungsdienst

4.12 Organisation OP

4.12.1 Operationssaal

4.12.2 OP-Planung und -Koordination

4.13 Sturz

4.13.1 Sturzprophylaxe

4.13.2 Intervention nach einem Sturz

Tabelle 2

Behandlung: «Schmerztherapie».

\section{Präventionsmassnahme}

Jeder Patient mit Schmerzen im OEB wird im Hinblick auf die Art und Intensität der Beschwerden eingeschätzt. Jeder Mitarbeiter ist mit einer Visuellen Analogskala (VAS) ausgestattet und im Umgang damit geschult.

Das Schmerzmanagementkonzept ist unter zentraler Leitung in der Klinik disziplinübergreifend abgestimmt.

Es stehen u.a. 4 PCA-Pumpen zur Verfügung. Diese Ausstattung ist nach Auskunft der Interviewpartner ausreichend.

Es existiert ein Standard zur postoperativen Analgesie. Auf jeder der Stationen ist eine «Schmerzbox» untergebracht. Neben der Medikamentenliste befindet sich darin das Analgesiekonzept in Schriftform sowie Erstanamnesebögen und Schmerzprotokolle. Der Standard ist offiziell per Unterschrift des verantwortlichen Arztes freigegeben.

Eine Schmerzmittelapplikation durch Mitarbeiter des Pflegedienstes ist immer zuvor vom behandelnden Arzt angeordnet. Die Mitarbeiter des Pflegedienstes sind nachweislich autorisiert, Opiate in definiertem Rahmen zu applizieren.

Sämtliche Schmerzmittelgaben werden in der Verlaufsdokumentation eingetragen.

Die anordnende Person sowie die verabreichende Person des Teams sind retrospektiv identifizierbar.
Risiko

Empfehlung 
Tabelle 3

Dokumentation: «Geburtsverlauf».

\section{Präventionsmassnahme}

Im Kreisssaal kommt ein auf die abteilungsinternen Besonderheiten abgestimmtes Dokumentationssystem zur Anwendung. Die präpartalen Kontrollen, der Geburtsverlauf, die Entbindung (inkl. aller notwendigen Informationen) und die kindlichen Daten (Apgar-Score etc.) werden auf dem Partogramm dokumentiert. Vitalzeichen und alle anderen Aktivitäten werden in definierten Abständen erfasst und im Partogramm/Verlauf dokumentiert.

Zusätzliche Patientendaten werden in eine EDV-Dokumentation eingegeben. In dem Phoenix-Programm werden alle, die stationäre Betreuung betreffenden Daten erfasst. Hiermit dokumentieren Arzt, Hebamme und Pflege gemeinsam.

Im Geburtsverlauf/Partogramm werden sämtliche Daten, Untersuchungsbefunde, Tätigkeiten und Befindlichkeiten der Patientinnen von der Kreisssaalaufnahme bis zu Verlegung dokumentiert.

Vorbefunde aus dem Mutterpass werden zur Kenntnis genommen und relevante Daten (z.B. Parität, Grav., Erkrankungen der Schwangerschaft etc.) in die geburtshilfliche Dokumentation übertragen. Diese Tätigkeit wird von der diensthabenden Hebamme durchgeführt.

Die Zuständigkeit für die Kontrolle und den Übertrag von Vorbefunden ist eindeutig geregelt.

Es kommt ein spezielles Überwachungsprotokoll bei Patientinnen zum Einsatz, die mit einer Periduralanästhesie (PDA) versorgt werden (Anlage, Medikamentengabe, Vitalzeichenkontrolle). Dokumentiert werden die Anlage der PDA und die Vitalzeichen im Anästhesieprotokoll und im Partogramm.

\section{Risiko}

Empfehlung

Arzt und Hebamme dokumentieren nicht gemeinsam in der Kreisssaaldokumentation Die geburtshilfliche Dokumentation erfolgt ausschliesslich durch die Hebamme. Der Arzt dokumentiert sein Tätigwerden nicht im Partogramm oder Verlauf.

Ein Personalwechsel wird im Partogramm nicht zwingend mit Namensnennung der dienstübernehmenden Hebamme dokumentiert. Auf dem Partogramm ist lediglich vorgesehen, dass die Hebamme, welche die Geburt begleitet, eine Unterschrift leistet. Der Name der Hebamme, die die Schwangere aufnimmt, wird somit nicht immer erfasst.
Wird der Arzt von der Hebamme hinzugezogen und muss er tätig werden oder Anordnungen geben, sollte er diese im Partogramm/Verlauf dokumentieren.

Stand: 10.10 .2005

- wird umgesetzt

Bei Aufnahme einer Schwangeren in den Kreisssaal sollte die Hebamme mit Datum und Uhrzeit die Aufnahme dokumentieren.

Stand: 10.10.2005

- wird umgesetzt 
Tabelle 4

Patientenaufklärung: «Allgemeine Patientenaufklärung» (Ausschnitt).

\section{Präventionsmassnahme}

Das Prozedere im Zusammenhang mit der Aufklärung ist abteilungsintern festgelegt.

Zur Dokumentation der Aufklärungsgespräche werden Aufklärungsbögen der jeweiligen Fachgesellschaften genutzt.
Die Einwilligungsbögen werden mit handschriftlichen Ergänzungen bzw. Zeichnungen versehen.

Anhand der ausgefüllten Einwilligungsbögen ist ein individuell geführtes Aufklärungsgespräch nachweisbar.

Einwilligungsbögen werden nach dem Aufklärungsgespräch immer mit dem Datum, der Uhrzeit und der Unterschrift des Arztes sowie des Patienten versehen.

Anhand der ausgefüllten Einwilligungsbögen ist ein individuell geführtes Aufklärungsgespräch nachweisbar
Risiko

Die Formulare liegen nicht immer in der aktuellen Version vor.

\section{Empfehlung}

Das aktuelle Aufklärungsformular sollte genutzt werden.

Standardisierte Einwilligungsbögen eignen sich ebenfalls zur Dokumentation des Aufklärungsgesprächs. Insbesondere die plastischen Abbildungen der Operationen erleichtert es den Patienten, die operative Vorgehensweise zu verstehen.

Stand: 10.10.2005:

- mit Einführung der elektronischen Version der Aufklärungsformulare ist die Aktualität gewährleistet

In der Unfallchirurgie wird derzeit ein Aufklärungsprotokoll/Einwilligung der Schweizerischen Gesellschaft für Chirurgie aus dem Jahr 1997 verwendet, in dem keine Gegenzeichnung durch den Patienten erfolgt.
Eine entsprechende Dokumentation sollte erfolgen.

Stand 10.10.2005:

- erledigt

\section{Tabelle 5}

Organisation: «Patientenaufnahme und -entlassung» (Ausschnitt).

\section{Präventionsmassnahme}

Im OEB findet täglich eine Aufnahme- und Entlassungsplanung statt. Die Aufnahme- und Entlassungsplanung richtet sich nach den Kapazitäten an den OP-Tagen.

Bei der Aufnahmeplanung findet eine Abstimmung zwischen Operations-und ggf. Bettenkapazitäten statt. Mit dem OP-Buch wird die OP-Kapazität und die daraus resultierenden Aufnahmen abgestimmt.

Bei jedem Patienten wird im Rahmen der Aufnahme eine ärztliche und pflegerische Anamnese erstellt.

Beide Berufsgruppen stellen die erhobenen Anamnesen in das Phoenix-Programm ein.

Die Befunde aus dem Erstkontakt und die Aufnahmediagnose werden am gleichen Tag evaluiert, d. h. einem Facharzt bereits im Rahmen der Notaufnahme vorgestellt. In der Mittagsbesprechung werden die Patienten, die aufgenommen wurden, dem Chirurgen und dem Anästhesisten vorgestellt.

Die Ergebnisse der Anamneseerhebung werden strukturiert und übersichtlich dokumentiert. Diese können von allen berechtigten Personen im Phoenix-Programm eingesehen werden.

Jeder Patient erhält einen Entlassungsarztbrief mit dem Behandlungsschema für den weiterbehandelnden Arzt.
Risiko

Empfehlung

Patienten erhalten bei der Entlassung kein Merkblatt mit spezifischen Verhaltensregeln
Die Entwicklung von Merkblättern mit spezifischen Verhaltensregeln, die dem Patienten ausgehändigt werden können, sollte mit der Weiterentwicklung von Behandlungsstandards einhergehen.

Stand 10.10.2005:

- wird mit in das neue Entlassmanagement integriert 